\title{
DIRECT DECOMPOSITIONS OF ALGEBRAIC SYSTEMS ${ }^{1}$
}

\author{
BY PETER CRAWLEY AND BJARNI JÓNSSON \\ Communicated by Leon Henkin, February 19, 1963
}

Preliminaries. An operator group with a principal series can obviously be written as a direct product of finitely many directly indecomposable admissible subgroups, and the classical WedderburnRemak-Krull-Schmidt theorem asserts that this representation is unique up to isomorphism. Numerous generalizations of this theorem are known in the literature. ${ }^{2}$ Thus it follows from the results in Baer $[1 ; 2]$ that if the admissible center of an operator group $G$ satisfies the minimal and local maximal conditions, then any two direct decompositions of $G$ (with arbitrarily many factors) have isomorphic refinements. In a somewhat different direction, it is shown in Crawley [3] that if an operator group $G$ has a direct decomposition each factor of which has a principal series, then any two direct decompositions of $G$ have isomorphic refinements. The results announced here yield sufficient conditions for a group (with or without operators) to have the isomorphic refinement property, one consequence being a common generalization of the two theorems just mentioned. A more detailed treatment, giving proofs, will appear shortly in [4].

Actually our results hold for a much wider class of algebraic systems, and it is in this more general framework that the theory is developed. The terminology to follow is largely the same as that in Jónsson-Tarski [6], and therefore it will be described only briefly. By an algebra we shall mean a system consisting of a set $A$, a binary operation + called addition, a distinguished element 0 called the zero element of the algebra, and operations $F_{t}(t \in T)$ each of which is of some finite rank $\rho(t)$, subject only to the following conditions: (i) $A$ is closed under the operation + and the operations $F_{t}(t \in T)$; (ii) for all $x \in A, x+0=0+x=x$; (iii) for all $t \in T, F_{t}(0, \cdots, 0)=0$. The set $T$ and the function $\rho$ are assumed to be the same for all algebras under consideration. We shall identify an algebra with the set of all its elements, and shall use the same symbols,,$+ F_{t}$, and 0 , to denote the operations and the zero elements of all the algebras. If no auxiliary operations $F_{t}$ are present, i.e. if $T=\phi$, then we refer to $A$ as a binary algebra. An obvious example of an algebra is an operator group, i.e. an algebra for which addition is associative, each element has an

1 This work was partially supported by National Science Foundation grants NSF G-17957 and NSF G-19673.

${ }^{2} \mathrm{~A}$ fairly complete list of references is given in Baer $[1 ; 2]$ and Specht $[8, \mathrm{p} .449]$. 
additive inverse, and each $F_{t}(t \in T)$ is a unary operation which distributes with respect to + . Similarly, an ordinary group without operators is a binary algebra.

The notions of subalgebra and isomorphism are assumed to be known. A subalgebra $B$ of an algebra $A$ is called a subtractive subalgebra if it satisfies the following condition: if $a \in A$ and $b \in B$, and if either $a+b \in B$ or $b+a \in B$, then $a \in B$. The subtractive subalgebras of an operator group are precisely its admissible subgroups.

By a central subalgebra of an algebra $A$ we mean a subalgebra $C$ of $A$ satisfying the following conditions: (i) for each $c \in C$ there exists $\bar{c} \in C$ such that $c+\bar{c}=0$; (ii) if $c \in C$ and $x, y \in A$, then $x+(y+c)$ $=(x+c)+y=(x+y)+c$; (iii) if $c \in C, t \in T, k<\rho(t)$, and $x_{0}, \cdots, x_{\rho(t)-1}$ $\in A$, then

$$
\begin{aligned}
& F_{t}\left(x_{0}, \cdots, x_{k-1}, x_{k}+c, x_{k+1}, \cdots, x_{\rho(t)-1}\right) \\
& \quad=F_{t}\left(x_{0}, \cdots, x_{\rho(t)-1}\right)+F_{t}(0, \cdots, 0, c, 0, \cdots, 0) .
\end{aligned}
$$

It is easy to see that the subalgebra generated by the union of all the central subalgebras of an algebra $A$ is again a central subalgebra of $A$; this largest central subalgebra is called the center of $A$, and is denoted by $A^{0}$. It is clear that if $A$ is an operator group, then $A^{\circ}$ is just the usual group-theoretic admissible center of $A$. More generally, for any algebra $A$, the set $A^{\circ}$ together with the operation + forms an abelian group. Two subalgebras $B$ and $C$ of $A$ are called centrally isomorphic if there is an isomorphism $f$ of $B$ onto $C$ such that for each $x \in B$ there is an element $c \in A^{c}$ with $f(x)=x+c$.

The concept of an algebra is designed to make it possible to define internally the notion of (restricted) direct product, analogous to the definition of direct product for groups. An algebra $A$ is a direct product of subalgebras $B_{i}(i \in I)$, in symbols

$$
A=\prod_{i \in I} B_{i},
$$

if the following conditions are satisfied:

(I) For each index $h \in I$, if $x, y \in B_{h}$ and $u$, $v$ are elements of the subalgebra generated by the union of the $B_{i}(i \neq h)$, then

$$
(x+u)+(y+v)=(x+y)+(u+v) .
$$

Notice that (I) implies that if $i_{0}, \cdots, i_{n-1}$ are finitely many distinct indices in $I$ and $x_{m} \in B_{i_{m}}(m=0, \cdots, n-1)$, then the elements $x_{0}, \cdots, x_{n-1}$ commute and associate with each other, and therefore the sum $x_{0}+\cdots+x_{n-1}$ is unambiguously defined.

(II) For every $h \in I$ and $t \in T$, if $x_{0}, \cdots, x_{\rho(t)-1} \in B_{h}$ and $y_{0}, \cdots$, 
$y_{\rho(t)-1}$ are elements of the subalgebra generated by the union of the $B_{i}(i \neq h)$, then

$$
\begin{aligned}
F_{t}\left(x_{0}+y_{0}, \cdots, x_{\rho(t)-1}\right. & \left.+y_{\rho(t)-1}\right) \\
& =F_{t}\left(x_{0}, \cdots, x_{\rho(t)-1}\right)+F_{t}\left(y_{0}, \cdots, y_{\rho(t)-1}\right) .
\end{aligned}
$$

(III) For each $a \in A$ there exist finitely many distinct indices $i_{0}, \cdots, i_{n-1} \in I$ and nonzero elements $x_{m} \in B_{i_{m}}(m=0, \cdots, n-1)$ such that

$$
a=x_{0}+\cdots+x_{n-1},
$$

and this representation (apart from the order of the summands) is unique.

The direct product of finitely many subalgebras $B_{0}, \cdots, B_{n-1}$ of an algebra $A$ will also be written $B_{0} \times \cdots \times B_{n-1}$. A subalgebra $B$ of $A$ is called a factor of $A$ if $A=B \times C$ for some subalgebra $C$. An algebra $A$ is indecomposable if $A$ has at least two elements and the only factors of $A$ are $A$ itself and $\{0\}$. A representation of an algebra $A$ as a direct product of subalgebras is called a direct decomposition of $A$. Two direct decompositions of $A$,

$$
A=\prod_{i \in I} B_{i}=\prod_{j \in J} C_{j}
$$

are said to be (centrally) isomorphic if there is a one-to-one mapping $f$ of $I$ onto $J$ such that, for each $i \in I, B_{i}$ and $C_{f(i)}$ are (centrally) isomorphic. Finally, the second decomposition is said to be a refinement of the first if for each $j \in J$ there exists $i \in I$ such that $C_{j} \subseteq B_{i}$.

The exchange and isomorphic refinement properties. Our techniques are based on an exchange property defined as follows. An algebra $B$ has the exchange property if for any algebra $A$ containing $B$ as a subalgebra, and for any subalgebras $C$ and $D_{i}(i \in I)$ of $A$, the condition

$$
A=B \times C=\prod_{i \in I} D_{i}
$$

implies that there exist subalgebras $E_{i} \subseteq D_{i}(i \in I)$ such that

$$
A=B \times \prod_{i \in I} E_{i} \text {. }
$$

The following lemma is fundamental: if $n$ is a positive integer, and if $B_{0}, \cdots, B_{n}$ are subalgebras of an algebra $B$ with $B=B_{0} \times \cdots \times B_{n}$, then $B$ has the exchange property if and only if each of the factors $B_{m}(m=0, \cdots, n)$ has the exchange property. It should be noted that this lemma fails for infinite products; there are simple examples of 
abelian groups which do not have the exchange property but are direct products of infinitely many subgroups each having the exchange property.

Consider now the central question of this paper: is it true that if an algebra $A$ is a direct product of subalgebras each of which has the exchange property, then any two direct decompositions of $A$ have isomorphic refinements? In general this question remains open. However, the following theorems provide affirmative answers in three special cases.

(A) If an algebra $A$ has two direct decompositions with countably many factors,

$$
A=B_{0} \times B_{1} \times B_{2} \times \cdots=C_{0} \times C_{1} \times C_{2} \times \cdots,
$$

where all the factors $B_{i}$ and $C_{j}$ have the exchange property, then these two direct decompositions have centrally isomorphic refinements.

(B) If an algebra $A$ is a direct product of subalgebras each of which has the exchange property, then any two direct decompositions of $A$ with indecomposable factors are centrally isomorphic.

(C) If an algebra $A$ is a direct product of subalgebras each of which has the exchange property and has a countably generated center, then any two direct decompositions of $A$ have centrally isomorphic refinements.

Conditions that imply the exchange property. Theorems (A), (B) and $(C)$, which relate the exchange and isomorphic refinement properties, lead us to seek conditions on an algebra $B$ for it to have the exchange property. The following simple result reduces this to the center: if the center $B^{\circ}$ of an algebra $B$ has the exchange property, then $B$ has the exchange property. A further reduction is possible, which reduces the problem to one essentially involving only abelian operator groups. Define an algebra $A$ to be abelian if $A$ coincides with its center, $A=A^{\circ}$.

(D) In order for an algebra $B$ to have the exchange property it is sufficient that the following condition be satisfied: for any abelian algebra $A$ containing $B^{c}$ as a subalgebra, and for any subalgebras $C$ and $D_{i}(i \in I)$ of $A$, if

$$
A=B^{\circ} \times C=\prod_{i \in I} D_{i},
$$

and if each of the subalgebras $D_{i}(i \in I)$ is isomorphic to a subalgebra of $B^{c}$, then there exist subalgebras $E_{i} \subseteq D_{i}(i \in I)$ such that

$$
A=B^{\bullet} \times \prod_{i \in I} E_{i} \text {. }
$$


Using (D) in conjunction with results in Baer [1] we obtain a structural condition on the center of an algebra $B$ that implies that $B$ has the exchange property. This condition involves the following chain conditions. An algebra $A$ is said to satisfy the minimal (maximal) condition if every nonempty family of subtractive subalgebras of $A$ has a minimal (maximal) member. An algebra $A$ satisfies the local maximal condition if every finitely generated subtractive subalgebra satisfies the maximal condition. When specialized to operator groups, these conditions are just the usual group-theoretic chain conditions.

(E) If the center $B^{\circ}$ of an algebra $B$ satisfies the minimal and local maximal conditions, then $B$ has the exchange property, and $B^{c}$ is countably generated.

Combining (C) and (E) we obtain our principal isomorphic refinement theorem for general algebras.

(F) If an algebra $A$ has a direct decomposition

$$
A=\prod_{i \in I} B_{i}
$$

such that, for each $i \in I, B_{i}^{c}$ satisfies the minimal condition and the local maximal condition, then any two direct decompositions of $A$ have centrally isomorphic refinements.

Binary algebras. When applied to algebras without auxiliary operations $F_{t}$, Theorem $(\mathrm{F})$ can be stated in the following equivalent form: if a binary algebra $A$ is a direct product of subalgebras $B_{i}(i \in I)$ such that, for each $i \in I, B_{i}^{c}$ is a direct product of finitely many primary cyclic groups and primary quasi-cyclic groups, then any two direct decompositions of $A$ have centrally isomorphic refinements. For every abelian group satisfies the local maximal condition, and the condition imposed on the abelian groups $B_{i}^{c}$ above is equivalent to the minimal condition. It is possible, however, to obtain considerably more general results than the one just stated, results that eliminate chain conditions altogether. For their formulation, some concepts from the theory of abelian groups are needed. ${ }^{3}$

If $G$ is an abelian group and $n$ is an integer, then the subgroup $n G$ is defined as usual by $n G=\{n x \mid x \in G\}$. An abelian group $G$ is divisible if $n G=G$ for all $n \neq 0$, and $G$ is of bounded order if there is an integer $n \neq 0$ such that $n G=\{0\}$. Every abelian group $G$ contains a maximal divisible subgroup $D$, which is a factor of $G$, and the quotient group $G / D$ is called the reduced part of $G$.

Let $G$ be an abelian $p$-group ( $p$ some prime). A subgroup $U$ of $G$

\footnotetext{
${ }^{3}$ For a detailed treatment see Fuchs [5] or Kaplansky [7].
} 
is called a basic subgroup if $U$ has the following properties: (i) $U$ is a direct product of cyclic groups; (ii) $U$ is a pure subgroup of $G$, i.e., $U \cap p^{n} G=p^{n} U$ for all $n>0$; (iii) the quotient group $G / U$ is divisible. Every abelian $p$-group contains at least one basic subgroup, and all the basic subgroups of a given group are isomorphic.

An abelian $p$-group $G$ is said to have no elements of infinite height if $\bigcap_{n<\infty} p^{n} G=\{0\}$. In such a group $G$ the $p$-adic topology can be introduced by taking as a neighborhood basis for 0 the subgroups $p^{n} G(n=1,2, \cdots)$. If an abelian $p$-group $G$ with no elements of infinite height has the property that every Cauchy sequence $\left\{x_{k}\right\}_{k<\infty}$ in $G$, for which the orders of the elements $x_{k}$ are bounded, converges to a limit in $G$, then $G$ is called torsion-complete.

An explicit representation of torsion-complete abelian p-groups can be given as follows. Let $U_{n}(n=1,2, \cdots)$ be a sequence of groups such that $U_{n}$ is a direct product of cyclic groups of order $p^{n}$. Let $\Gamma$ be the Cartesian product of the $U_{n}$ 's, that is, $\Gamma$ is the set of all functions $f$ defined on the positive integers such that $f(n) \in U_{n}$, with addition defined component-wise. Then the torsion subgroup of $\Gamma$ is a torsion-complete abelian $p$-group. Conversely, if $G$ is any torsioncomplete abelian $p$-group, then there exists a sequence of groups $U_{n}(n=1,2, \cdots)$, where $U_{n}$ is a direct product of cyclic groups of order $p^{n}$, such that $G$ is isomorphic to the torsion subgroup of the Cartesian product of the $U_{n}$ 's. In particular, every primary abelian group of bounded order is torsion-complete, and every countable torsion-complete primary abelian group is necessarily of bounded order.

(G) If $B$ is a binary algebra such that the reduced part of $B^{c}$ is a torsion group each primary component of which is torsion-complete, then $B$ has the exchange property.

Combining $(\mathrm{G})$ with $(\mathrm{A}),(\mathrm{B})$ and $(\mathrm{C})$, respectively, we obtain the following isomorphic refinement theorems.

(H) If $A$ is a binary algebra such that the reduced part of $A^{c}$ is a torsion group with torsion-complete primary components, then any two countable direct decompositions of $A$ have centrally isomorphic refinements.

(I) If a binary algebra $A$ has a direct decomposition

$$
A=\prod_{i \in I} B_{i}
$$

where, for each $i \in I$, the reduced part of $B_{i}^{c}$ is a torsion group with torsion-complete primary components, then any two direct decompositions of $A$ into indecomposable factors are centrally isomorphic. 
(J) If a binary algebra $A$ has a direct decomposition

$$
A=\prod_{i \in I} B_{i}
$$

where, for each $i \in I, B_{i}^{c}$ is countable and the reduced part of $B_{i}^{c}$ is a torsion group each primary component of which is of bounded order, then any two direct decompositions of $A$ have centrally isomorphic refinenents.

A final theorem describes a class of binary algebras with uncountable centers having the isomorphic refinement property.

(K) If $A$ is a binary algebra such that the maximal divisible subgroup of $A^{c}$ is countable and the reduced part of $A^{c}$ is a torsion group each primary component of which is a torsion-complete group with countable basic subgroups, then any two direct decompositions of $A$ have centrally isomorphic refinements.

\section{REFERENCES}

1. R. Baer, Direct decompositions, Trans. Amer. Math. Soc. 62 (1947), 62-98.

2. - Direct decompositions into infinitely many summands, Trans. Amer. Math. Soc. 64 (1948), 519-551.

3. P. Crawley, Direct decompositions with finite dimensional factors, Pacific J. Math. 12 (1962), 457-468.

4. P. Crawley and B. J6nsson, Refinements for infinite direct decompositions of algebraic systems, (to appear).

5. L. Fuchs, Abelian groups, Budapest, 1958.

6. B. Jonsson and A. Tarski, Direct decompositions of finite algebraic systems, Notre Dame Mathematical Lectures, no. 5, 1947.

7. I. Kaplansky, Infinite abelian groups, Univ. of Michigan Press, Ann Arbor, Mich., 1956.

8. W. Specht, Gruppentheorie, Springer, Berlin, 1956.

University of California, Berkeley and UNIVERSITY OF MiNNESOTA 